\title{
Safety and efficacy of cognitive training plus epigallocatechin-3-gallate in young adults with Down's syndrome (TESDAD): a double-blind, randomised, placebo-controlled, phase 2 trial
}

Rafael de la Torre, Susana de Sola, Gimena Hernandez, Magí Farré, Jesus Pujol, Joan Rodriguez, Josep María Espadaler, Klaus Langohr, Aida Cuenca-Royo, Alessandro Principe, Laura Xicota, Nathalie Janel, Silvina Catuara-Solarz, Gonzalo Sanchez-Benavides, Henri Bléhaut, Iván Dueñas-Espín, Laura del Hoyo, Bessy Benejam, Laura Blanco-Hinojo, Sebastiá Videla, Montserrat Fitó, Jean Maurice Delabar, Mara Dierssen for the TESDAD study group

\section{Summary}

Background Early cognitive intervention is the only routine therapeutic approach used for amelioration of intellectual deficits in individuals with Down's syndrome, but its effects are limited. We hypothesised that administration of a green tea extract containing epigallocatechin-3-gallate (EGCG) would improve the effects of non-pharmacological cognitive rehabilitation in young adults with Down's syndrome.

Methods We enrolled adults (aged 16-34 years) with Down's syndrome from outpatient settings in Catalonia, Spain, with any of the Down's syndrome genetic variations (trisomy 21, partial trisomy, mosaic, or translocation) in a double-blind, placebo-controlled, phase 2, single centre trial (TESDAD). Participants were randomly assigned at the IMIM-Hospital del Mar Medical Research Institute to receive EGCG ( $9 \mathrm{mg} / \mathrm{kg}$ per day) or placebo and cognitive training for $12 \mathrm{months}$. We followed up participants for 6 months after treatment discontinuation. We randomly assigned participants using randomnumber tables and balanced allocation by sex and intellectual quotient. Participants, families, and researchers assessing the participants were masked to treatment allocation. The primary endpoint was cognitive improvement assessed by neuropsychologists with a battery of cognitive tests for episodic memory, executive function, and functional measurements. Analysis was on an intention-to-treat basis. This trial is registered with ClinicalTrials.gov, number NCT01699711.

Findings The study was done between June 5, 2012, and June 6, 2014. 84 of 87 participants with Down's syndrome were included in the intention-to-treat analysis at 12 months (43 in the EGCG and cognitive training group and 41 in the placebo and cognitive training group). Differences between the groups were not significant on 13 of 15 tests in the TESDAD battery and eight of nine adaptive skills in the Adaptive Behavior Assessment System II (ABAS-II). At 12 months, participants treated with EGCG and cognitive training had significantly higher scores in visual recognition memory (Pattern Recognition Memory test immediate recall, adjusted mean difference: $6 \cdot 23$ percentage points [95\% CI 0.31 to $12 \cdot 14$ ], $\mathrm{p}=\mathbf{0} \cdot 039 ; d \mathbf{0} \cdot 4$ [0.05 to 0.84 ]), inhibitory control (Cats and Dogs total score, adjusted mean difference: 0.48 [0.02 to 0.93$], \mathrm{p}=0.041 ; d 0.28[0.19$ to 0.74$]$; Cats and Dogs total response time, adjusted mean difference: $-4.58 \mathrm{~s}[-8.54$ to -0.62$], \mathrm{p}=0.024 ; d-0 \cdot 27[-0.72$ to $-0 \cdot 20]$ ), and adaptive behaviour (ABAS-II functional academics score, adjusted mean difference: $5 \cdot 49[2 \cdot 13$ to $8 \cdot 86], \mathrm{p}=0 \cdot 002 ; d 0 \cdot 39[-0 \cdot 06$ to $0 \cdot 84])$. No differences were noted in adverse effects between the two treatment groups.

Interpretation EGCG and cognitive training for 12 months was significantly more effective than placebo and cognitive training at improving visual recognition memory, inhibitory control, and adaptive behaviour. Phase 3 trials with a larger population of individuals with Down's syndrome will be needed to assess and confirm the long-term efficacy of EGCG and cognitive training.

Funding Jérôme Lejeune Foundation, Instituto de Salud Carlos III FEDER, MINECO, Generalitat de Catalunya.

\section{Introduction}

Down's syndrome is the most common intellectual disability of genetic origin, affecting more than 5 million people worldwide. Medical interventions have substantially increased the life expectancy of individuals with Down's syndrome in high-income countries, but cognitive and behavioural features have a strong effect on functional ability. No approved intervention exists for the amelioration of cognitive deficits in individuals with Down's syndrome. ${ }^{1,2}$ Only early intervention programmes have resulted in some improvement in intellectual disability. ${ }^{3}$ Also, memory-training programmes have been reported to improve memory in adults with Down's syndrome. ${ }^{4}$

Evidence suggests that flavonoid-rich foods (eg, green tea) can improve normal cognitive function and might
Lancet Neurol 2016 Published Online May 20, 2016 http://dx.doi.org/10.1016/ S1474-4422(16)30034-5 See Online/Comment http://dx.doi.org/10.1016/ S1474-4422(16)30056-4 IMIM-Hospital del Mar Medical Research Institute and CIBER of Physiopathology of Obesity and Nutrition (CIBEROBN), University Pompeu Fabra (CEXS-UPF), Barcelona, Spain (R de la Torre PharmD, S de Sola PhD, M Farré MD, J Rodriguez MSc, A Cuenca-Royo PhD, L Xicota MSc, G Hernandez MD,

G Sanchez-Benavides PhD, I Dueñas-Espín MD,

Ldel Hoyo MSc, M Fitó MD); Center for Genomic Regulation (CRG), The Barcelona Institute of Science and Technology, CIBER of Rare Diseases (CIBERER), and University Pompeu Fabra (CEXS-UPF), Barcelona, Spain

(SCatuara-Solarz BsC, Prof $M$ Dierssen MD); Autonomous University of Barcelona (UDIMAS-UAB), Barcelona, Spain (M Farré MD); MRI

Research Unit, Hospital del Mar Medical Research Institute and CIBER Mental Health (CIBERSAM G21), Barcelona, Spain (J Pujol MD, A Principe MD, L Blanco-Hinojo PhD); Neurofunctionality of Brain and Language Research Group-Neurosciences Program IMIM-Hospital del Mar Medical Research Institute, Barcelona, Spain (J M Espadaler MD); Polytechnic University of Catalonia, Barcelona, Spain (K Langohr PhD); University Paris Diderot, Sorbonne Paris Cité, Unité de Biologie Fonctionnelle et Adaptative, UMR 8251 CNRS, 
Paris, France (N Janel PhD, J M Delabar PhD); Inserm, U1127, CNRS, UMR 7225 ICM, 75013, Sorbonne Universités, UPMC Univ Paris 06, UMR S 1127, Institut du Cerveau et de la Moelle épinière, ICM, F-75013, Paris, France (J M Delabar); Jérôme Lejeune Foundation, Paris, France (H Bléhaut MD); Fundació Catalana Síndrome de

Down, Barcelona, Spain (B Benejam BsC, S Videla MD); and Universitat Pompeu Fabra (DCEXS-UPF), Barcelona, Spain (SVidela)

Correspondence to:

Prof Mara Dierssen, Systems Biology Program, CRG-Center for Genomic Regulation,

c/ Dr Aiguader, 88 PRBB Building, 08003 Barcelona, Spain mara.dierssen@crg.eu

\section{Research in context}

\section{Evidence before this study}

We searched PubMed for reports published from Jan 1, 1995, to Dec 31, 2015 in English, with the terms "Down syndrome", "trisomy 21", "supplements", "drugs", and "cognitive treatment" or "cognition therapy". We included all randomised clinical trials of dietary supplements and drugs reporting any assessment of cognitive function in patients with Down's syndrome. Apart from the pilot clinical trial by our study group in 2010, we identified six additional clinical trials with four different interventions: three with donepezil, one with folinic acid, vitamins, and minerals, one with memantine, and one with L-acetyl carnitine.

\section{Added value of this study}

Cognitive impairments associated with intellectual disability syndromes were believed to be intractable, but recent progress in our understanding of the mechanisms underlying the impairments associated with genetic syndromes, such as fragile $X$ syndrome and tuberous sclerosis, has resulted in identification of potential treatment targets, which are being tested in clinical trials. It is increasingly argued that even complex syndromes such as Down's syndrome are potentially treatable. Most therapies targeting cognition in Down's syndrome are neurotransmitter based, and are more often used in treating Alzheimer's disease, like acetylcholinesterase inhibitors (donezepil, rivastagmine, galantamine), GABAergic antagonists (eg, pentetrazol), and N-methyl-D-aspartate receptor antagonists (memantine). Additionally, other compounds such as vitamins, mineral supplements, piracetam, or growth hormone have been used with little or no success. None of the previous clinical trials had combined pharmacological treatment with cognitive training and none of them showed significant improvement in cognition, adaptive functionality, or language performance. This is the first randomised controlled clinical trial using a dietary supplement (green tea extracts containing epigallocatechin-3-gallate [EGCG], a green tea catechin), combined with cognitive training. Our study shows a significant improvement in memory, executive function, and facilitated adaptive behaviour. A major strength of the trial is the inclusion of secondary outcome measures, such as neuroimaging and neurophysiology complementary explorations. Our findings suggest that the treatment had a measurable effect on biomarkers such as cholesterol and homocysteine. Additionally, neuroimaging showed increased functional connectivity and normalised cortical excitability. Furthermore, the treatment resulted in few side-effects.

\section{Implications of all the available evidence}

Our findings suggest the possibility of improving long-term outcomes in individuals with Down's syndrome, and also open the way for clinical trials of other treatments in this population, such as more specific or more potent DYRK1A inhibitors (already in development), antioxidants, or lipid modulators. EGCG and cognitive training are easy-to-use, affordable, and widely accessable. This approach could lead to clinically validated interventions manageable at the primary care level. Effective treatments will have substantial implications for health services because even small clinical improvements in cognitive outcomes could lead to substantial reductions in lifetime care needs for individuals with intellectual disability syndromes. have therapeutic effects in Alzheimer's disease because 3 their biological actions include antioxidant effects; they also have the potential to protect susceptible neurons, enhance existing neuronal function, reduce toxic concentrations of amyloid $(A \beta)$, and stimulate neuronal plasticity. ${ }^{1,5}$ Bain and colleagues ${ }^{6}$ described the noncompetitive inhibition properties of epigallocatechin-3gallate (EGCG), the major catechin in green tea leaves (40-50\% of total catechins) on the kinase activity of the dual-specificity tyrosine-(Y)-phosphorylation-regulated kinase 1A (DYRK1A), a serine-threonine kinase encoded by the DYRK1A gene, located in the Down's syndrome critical region (ie, on the long arm of chromosome 21) and thought to be a major contributor to cognitive phenotypes of Down's syndrome. EGCG can cross the blood-brain barrier, 7 and chronic administration of a green tea extract containing 45\% EGCG promoted learning and memory in Down's syndrome mouse models ${ }^{8}$ and was safe in young individuals with Down's syndrome in our phase 1 trial. ${ }^{9}$ Housing conditions that facilitate enhanced sensory, cognitive, motor, and social stimulation normalises DYRK1A kinase activity in the hippocampus of Down's syndrome mouse models, suggesting that EGCG acts synergistically with cognitive stimulation. ${ }^{10}$ The long-term efficacy of cognitive training has not been conclusively shown in individuals with Down's syndrome. In this phase 2 trial, we compared the effectiveness of cognitive training plus placebo with cognitive training plus EGCG in adults with Down's syndrome.

\section{Methods}

\section{Study design and participants}

The TESDAD study was a randomised, double-blind, placebo-controlled, phase 2 trial in adults with Down's syndrome, and was done at the Hospital del Mar Medical Research Institute of Barcelona (Spain).

The trial was approved by the local ethics committee (CEIC Parc de Salut Mar, EGCG/DYRK1A/DS/IMIM/1), and done according to the Declaration of Helsinki and Spanish guidelines and regulations for data privacy.

Eligible participants, whose parent or carer agreed to ensure administration of the medication and attendance at all visits, were enrolled from a large cohort of outpatients of the Catalan Down Syndrome Foundation (Barcelona, Spain). We planned to include adults aged 18-30 years of both sexes with Down's syndrome (genetic 
variations of trisomy 21, partial trisomy, mosaic, or 1 containing 45\% EGCG (Life Extension Decaffeinated translocation) but, because of difficulties in recruitment, we widened the age range for inclusion to 16-34 years. We included participants with a BMI between $18 \cdot 5 \mathrm{~kg} /$ $\mathrm{m}^{2}$ and $29.9 \mathrm{~kg} / \mathrm{m}^{2}$.

Patients with neurological disease other than Down's syndrome, relevant medical disease (ie, comorbid leukaemia or high-flow congenital heart disease), comorbid unstable mental disorder, or taking any treatment that could interfere with cognitive function were excluded from the study. Other exclusion criteria were any major illness or major surgery in the past 3 months before participation in the study; new or irregular medication in the month preceding the study; ingestion of vitamin or catechin supplements or nonsteroidal anti-inflammatory drugs in the 2 weeks before the study; gastrointestinal, hepatic, renal, or any other problems that might alter absorption, distribution, metabolism, or excretion of the drug. During the run-in period, participants had a brief cognitive assessment to 20 estimate their intellectual disability based on the Diagnostic and Statistical Manual of Mental Disorders (DSM-IV).

Participants, parents, or their legal guardians were informed about the protocol and gave written informed consent.

\section{Randomisation and masking}

We randomly assigned participants to the EGCG and cognitive training group or the placebo and cognitive training group using random-number tables, and 30 allocation was balanced by sex and intellectual quotient (IQ) Wufman Brief Intelligence Test [K-BIT; appendix] pX] dividuals with an IQ of less than 40 were classified as having severe intellectual disability, whereas individuals with an IQ of 40-69 were classified as having moderate-tomild intellectual disability. This approach was taken to avoid bias in the inclusion of patients and an overrepresentation of either IQ category in one of the treatments groups. Sequence generation, allocation concealment with a sealed opaque envelope, treatment allocation, and provision of labelled packs (both packs with identical appearance and size zero blue opaque capsules) were done by the Hospital del Mar Pharmacy Department. To ensure that the capsules were consumed, the participants' carer or parent had to return the empty 45 medication (EGCG or placebo) vials to the study coordinator (JR) before receiving a new vial. All members of the research team, the statistician, participants with Down's syndrome, and their families or guardians were masked to treatment allocation. The double blind was 50 maintained until the end of the follow-up.

\section{Procedures}

Before treatment, both groups had 1 month of a singleblind placebo run-in period (participants and their families were masked). After the run-in period, participants received a green tea extract supplement

Mega Green Tea Extract; Life Extension, Fort Lauderdale, FL, USA) or placebo (rice flour). Each capsule contained $200 \mathrm{mg}$ of EGCG or rice flour. Participants weighing 5 50-75 kg were given three capsules (600 mg/day EGCG or placebo) and those weighing 75-100 kg were given four capsules ( $800 \mathrm{mg}$ /day EGCG or placebo) orally for 12 months, and had a follow-up visit 6 months after treatment discontinuation. All participants were to receive regular cognitive training sessions (30-50 min per session, 3 days per week) at home or at the offices of the Catalan Down Syndrome Foundation during the 12 months of treatment. The run-in period was to familiarise participants with the cognitive training programme software (FesKits) and routine. FesKits enables participants to access a wide range of training programmes and exercises on the internet. Cognitive training focused on memory, executive functions, and language and attention processes; training on memory comprised $50 \%$ of the content of each training session. Training was adapted to learning progression and a neuropsychologist did a weekly follow-up to ensure compliance with the cognitive training sessions. During the run-in period, while receiving placebo, patients attended five sham cognitive training sessions. After the run-in period, while also receiving medication in the double-blind phase, patients were recommended to perform three sessions per week during the 12 months of treatment.

At 6 months, an interim analysis was done to determine whether to continue based on adverse effects and early signs of efficacy.

\section{Outcomes}

The primary outcome was the change from baseline at 12 months in cognitive and functional components of the TESDAD neuropsychology battery, ${ }^{11}$ which included measures of attention, psychomotor speed, memory, executive functions, language, adaptive behaviour, quality of life, quality of sleep, and neuropsychiatric symptoms (appendix pXX@Aditionally, we screened for changes related to dementia onset with the Dementia Questionnaire for People with Intellectual Disabilities. The TESDAD battery was developed for testing cognitive performance and adaptive functionality in adults with Down's syndrome and was adapted from our phase 1 trial of EGCG.' All neuropsychological outcomes were assessed at baseline, and 3 months, 6 months, and 12 months after treatment initiation, and 6 months after treatment discontinuation. Since a gold standard does not exist for the assessment of cognition in individuals with intellectual disability, including those with Down's syndrome, we assessed the key cognitive and functional domains known to be impaired in patients with Down's syndrome and hypothesised to have clinical sensitivity to EGCG. A detailed description of the complete neuropsychological battery is provided in the appendix 
$(\mathrm{pXx}$ \& $y$ chomotor speed was measured with the Motor Screening Test (MOT, CANTAB). Attention was assessed with simple reaction time and span capacity measures using the Simple Reaction Time task (SRT, CANTAB); and the Spatial Span forward recall (SSP, CANTAB) and 5 the Digit Span forward recall from the Wechsler Adult Intelligence Scale-III (WAIS-III) were used to assess visual and verbal information, respectively. Measures of visual episodic memory and learning were obtained using the CANTAB Paired Associates Learning (PAL) and the 1 Pattern Recognition Memory test (PRM, CANTAB), and verbal episodic memory using the Cued Recall Test (CRT). For executive functioning, we assessed fractioned components of verbal fluency, working memory, planning, mental flexibility, and inhibitory control. Verbal 1 word fluency was measured with the semantic fluency word generation task (participants were asked to generate as many words as possible in $1 \mathrm{~min}$ belonging to the specified category of animals). Working memory for visual and verbal information was assessed with the SSP 20 (CANTAB) backward recall and the Digit Span backward recall (WAIS-III), respectively. Planning capacity was measured with the Tower of London from Drexel University (ToLDx) and mental flexibility with the Weigl Color-Form Sort Test. The Cats and Dogs Test was used to 2 assess response inhibition. Finally, measures of expressive and receptive language were obtained with the Boston Naming Test and the Token Test, respectively. Adult versions of the selected cognitive tests were used with the exception of four tests that were too complex for 3 adults with intellectual disability: the CRT (verbal episodic memory), the Cats and Dogs Test (inhibitory control), the Weigl Color-Form Sort Test (mental flexibility), and the ToLDX (planning ability). The child's versions of these tests were administered to avoid floor effects.

Everyday life functionality was assessed with questionnaires for the following domains: adaptive behaviour, quality of life, quality of sleep, and neuropsychiatric symptoms. Measures of adaptive behaviour were obtained with the adult version of the Adaptive Behavior Assessment System-Second Edition (ABAS-II). Quality of life was assessed with the parents' and guardians' version of the Kidscreen-27. Quality of sleep was assessed with the Pittsburgh Sleep Quality Index (PSQI) and neuropsychiatric symptoms were 4 assessed with the Neuropsychiatric Inventory (NPI).

We planned to monitor $A \beta-40$ and $A \beta-42$ peptides and their truncated forms as biomarkers of amyloidosis, related to Alzheimer's disease. Measurements were taken at baseline and at 6 months. However, we had 50 technical problems with the assay for plasma INNO-BIA $\mathrm{A} \beta$ forms (INNO-BIA; Fujirebio Europe, Gent, Belgium) and the results were consistent within but not among batches and therefore these measurements are not reported.

The secondary outcomes were the changes from baseline in the following biomarkers: plasma homocysteine and transthyretin concentrations (as measures of DYRK1A kinase activity); aspartate transaminase (AST) and alanine transaminase (ALT) concentrations (for safety); treatment compliance; and HDL, LDL, oxidised-LDL, total cholesterol, and triglycerides (to assess lipid oxidation).

We assessed adverse events, including serious adverse events, and vital signs, and did physical and neurological examinations, electrocardiogram (ECG), and standard laboratory assessments at each visit.

We also did exploratory analyses. We used functional MRI (fMRI) and transcranial magnetic stimulation (TMS) to measure the resting-state functional connectivity patterns with fMRI in a subsample of the participants due to financial constraints. Participants had three sessions of fMRI (at baseline, 6 months, and 12 months after treatment initiation). Our imaging approach was primarily based on whole-brain mapping of the degree of functional connectivity of each voxel with all other voxels 0 as the sum of correlations above a given Pearson's coefficient threshold. A subsequent region-of-interest mapping of functional connectivity was generated from the changes identified in the connectivity degn paps, using a seed-based approach ${ }^{12}$ (appendix pX $\Omega$ hree representative functional connectivity maps were obtained for each participant with regions of interest (seeds) placed at peak between-group connectivity degree differences in the frontal cortex, Wernicke's area, and cerebellum. Neurophysiological changes were assessed with paired pulse TMS. The TMS subsample included the same patients who had fMRI and were assessed at the same timepoints (baseline, 6 months, and 12 months after treatment initiation). Motor-evoked potentials (MEPs) were recorded from the right or left abductor 35 pollicis brevis muscle. The resting motor threshold (RMT) was set at the lowest stimulator output intensity capable of inducing an MEP of at least 50 microvolts peak-to-peak amplitude in relaxed state abductor pollicis brevis muscle in at least five of ten consecutive trials. Intracortical inhibition and data were obtained at interstimulus intervals of $3 \mathrm{~ms}$ and $5 \mathrm{~ms}$ (ICI-3 and ICI-5) and intracortical facilitation at interstimulus intervals of $10 \mathrm{~ms}$ and $15 \mathrm{~ms}$ (ICF-10 and ICF-15), with the conditioning stimulus set at an intensity of $80 \%$ of the RMT and the suprathreshold stimulus at $120 \%$ of the RMT. An average of ten trials was used to define the amplitudes of peak-to-peak MEPs. Other exploratory analyses were the assessment of stability or reversibility of changes at 18 months ( 6 months after discontinuation of interventions), and body composition (eg, weight, total fat mass, lean mass, total body water, BMI) with electrical impedance (TANITA-MC-180; Tanita Europe BV, Amsterdam, Netherlands).

The predefined rules for early termination of the study 55 were serious adverse events or negative results at the intermediate assessment of the study $(6$ months after randomisation). 


\section{Statistical analysis}

Sample size calculation was based on the results of the memory tests in our pilot study. ${ }^{9}$ We estimated that 100 individuals with Down's syndrome would have to be recruited to ensure 85 participants, assuming 15\% would discontinue. The size of the treatment difference was ten units with an SD of 18 units. To ensure a power of 0.85 using the $t$ test and assuming a mean difference of 10 units after 12 months of treatment with an SD of 15, 42 participants were needed in each group. 10 Participants excluded after the run-in period, before beginning treatment, were excluded from the analyses on the modified intention-to-treat population. The changes from baseline in scores on primary and secondary outcomes, including all tests scores and plasma biomarkers, were analysed using mixed models for repeated measures, and included treatment, time, treatment-by-time interaction, sex, and baseline scores as independent variables. The treatment-by-time interaction allowed examination of treatment differences over time, but was excluded whenever it was not significant. In these cases, the models assumed a constant treatment difference with respect to changes from baseline over time. Because missing data were generally less than $5 \%$, no imputation of missing values 25 was done. To analyse the differences between the two groups with respect to baseline after the washout period of 6 months, ANCOVA models were used for all test scores. These models used as outcome variables the changes from baseline to month 18 and included 30 treatment as an independent variable adjusting for sex and baseline score. The measure of interest in all the fitted regression models was the adjusted mean difference between both treatments with respect to changes from baseline. Additionally, the standardised 3 mean differences (Cohen's $d$ ) between both treatments were calculated for the changes from baseline after 12 months and 18 months. For the TMS assessments, because of the small subsample, the non-parametric Wilcoxon-Mann-Whitney test was used to study the 40 differences between treatments with respect to baseline. These additional analyses did not account for sex and baseline score. The fit of the linear mixed models and the ANCOVA models was done with the statistical software package $\mathrm{R}$ (version 3.1.1). Statistical 45 significance was set at $0 \cdot 05$. To protect against type II errors, no corrections were applied for multiple comparisons.

The trial is registered with ClinicalTrials.gov, number NCT01699711.

\section{Role of the funding source}

The funders of the study had no role in study design, data collection, data analysis, data interpretation, or writing of the report. The corresponding author had full 55 access to all the data in the study and had final responsibility for the decision to submit for publication.

\section{Results}

Between June 5, 2012, and June 6, 2014, we randomly assigned 87 patients ( $98 \%$ of 89 initially contacted). Three individuals withdrew before treatment initiation; 84 were included in the intention-to-treat analysis ( 41 in the placebo and cognitive training group and 43 in the EGCG and cognitive training group; figure 1). Demographic, genetic, and IQ characteristics were similarly distributed in both groups (table 1).

During the run-in period, patients were trained by neuropsychologists on how to do the cognitive training, but did not receive routine cognitive training. The total number of cognitive training sessions was similar between training groups (mean 107.5 [SD 48.8] vs 98.7 5 [46 -2] sessions). Mean number of sessions per week was 1.8 (SD 0.9) in the EGCG and cognitive training group and $2.0(0.9)$ in the placebo and cognitive training group; therefore, the compliance with cognitive training was suboptimum in both treatment groups (ie, less than the three recommended sessions per week). Differences between the groups were not significant on 13 of 15 tests in the TESDAD battery and eight of nine adaptive skills in the ABAS-II (appendix p 13-15).

In the EGCG plus cognitive training group after the

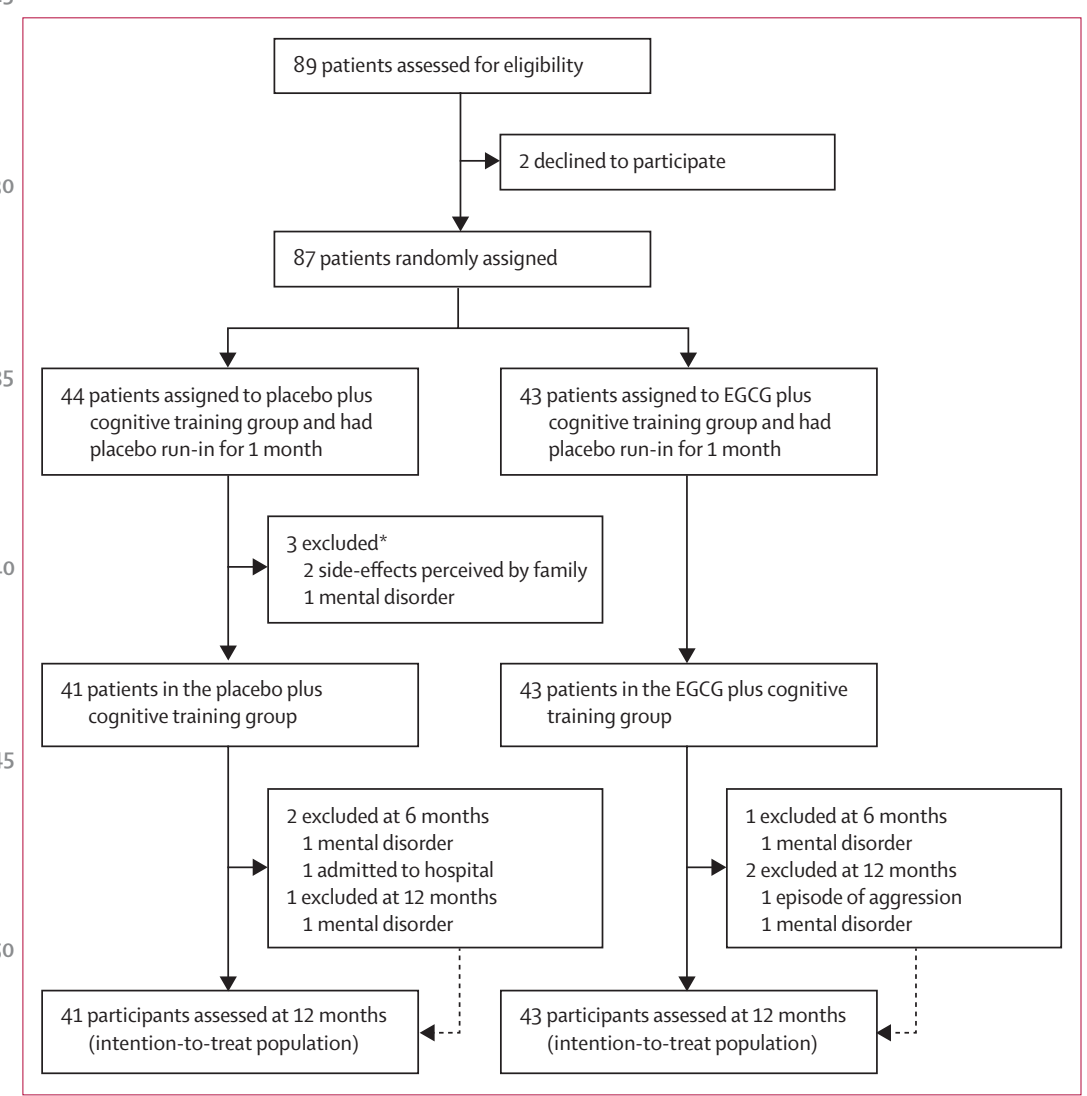

Figure 1: Trial profile

EGCG=epigallocatechin-3-gallate. fMRI=functional MRI. TMS=transcranial magnetic stimulation. *Withdrew before treatment in the placebo and cognitive training group and were not included in the intention-to-treat analysis. 


\begin{tabular}{|lcc|}
\hline & $\begin{array}{c}\text { Placebo plus cognitive } \\
\text { training group }(\mathrm{n}=44)\end{array}$ & $\begin{array}{c}\text { EGCG plus cognitive } \\
\text { training group }(\mathrm{n}=43)\end{array}$ \\
\hline Sex & $21(48 \%)$ & $24(56 \%)$ \\
Male & $23(52 \%)$ & $19(44 \%)$ \\
Female & $23 \cdot 4(4 \cdot 9)$ & $23 \cdot 1(3 \cdot 6)$ \\
Age (years) & & \\
Trisomy & $40(93 \%)$ \\
Simple & $43(98 \%)$ & $2(5 \%)$ \\
Translocation & 0 & 0 \\
Mosaicism & $1(2 \%)$ & $1(2 \%)$ \\
Partial trisomy & 0 & $25 \cdot 6(4 \cdot 2)$ \\
$\begin{array}{l}\text { Body-mass index } \\
\left.\text { (kg/m }{ }^{2}\right)\end{array}$ & $25 \cdot 9(4 \cdot 3)$ & $18(42 \%)$ \\
Intelligence quotient & & $25(58 \%)$ \\
$<40$ & $18(41 \%)$ & \\
$40-69$ & $26(59 \%)$ & \\
Data are number (\%) or mean (SD). EGCG=epigallocatechin-3-gallate. \\
\hline Table 1: Baseline demographic and clinical characteristics
\end{tabular}
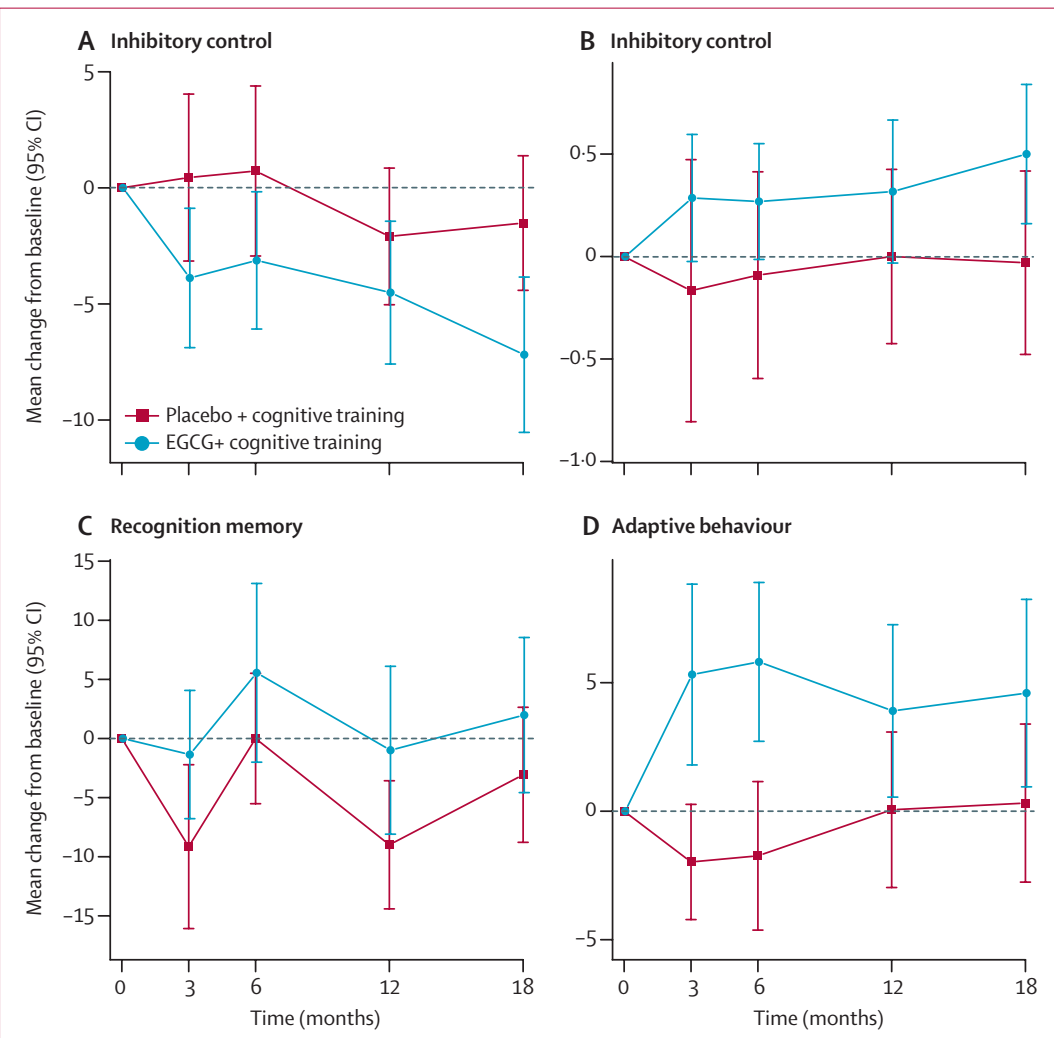

Figure 2: Effects of EGCG and cognitive training or placebo and cognitive training on neurocognitive performance and adaptive behaviour

Effects of EGCG and cognitive training versus placebo and cognitive training over time as assessed by the Cats and Dogs inhibition task, total time (A) and total score (B), the Pattern Recognition Memory (PRM) test, percent immediate recall (C), and adaptive behaviour in the Adaptive Behavior Assessment System II (ABAS-II) functional academics total score (D). EGCG=epigallocatechin-3-gallate.
112 months of treatment, we found a higher preservation of memory and an improvement of executive functioning compared with thenlegebo plus cognitive training group (figure 2, appendi: 1 ). At 12 months, the EGCG and 5 cognitive training group had a significantly lower decrease in the percentage of correct answers in the immediate visual memory recognition task with respect to baseline performance (PRM immediate recall; adjusted mean difference 6.23 percentage points 10 [95\% CI $0 \cdot 31$ to $12 \cdot 14], \mathrm{p}=0 \cdot 039 ; d 0 \cdot 4[0 \cdot 05$ to $0 \cdot 84])$ and better scores and reduced total response time in the inhibition task (Cats and Dogs total correct, adjusted mean difference: 0.48 [95\% CI 0.02 to 0.93 ], $\mathrm{p}=0 \cdot 041$; $d 0.28$ [0.19 to 0.74$]$; Cats and Dogs total response time, 15 adjusted mean difference: -4.58 s $[-8.54$ to $-0 \cdot 62]$, $\mathrm{p}=0 \cdot 024 ; d-0 \cdot 27[-0.72$ to $-0 \cdot 20])$ compared with the placebo and cognitive training group.

The EGCG and cognitive training group also showed improvement in adaptive behaviour in functional 0 academic skills, as measured with the ABAS-II functional academics score (adjusted mean difference 5.49 [95\% CI 2.13 to $8 \cdot 86$ ], $\mathrm{D}=0.002 ; d 0.39$ [ -0.06 to 0.84 ]; figure 2 , appendix pX Qut non-significant changes in social skills (ABAS socral skills, adjusted mean difference: $2 \cdot 56$ $25[-0 \cdot 22$ to $5 \cdot 35], \mathrm{p}=0 \cdot 071 ; d 0 \cdot 36[-0 \cdot 09$ to $0 \cdot 81])$ compared with the placebo and cognitive training group. Concerning quality of life, we found no significant difference among the groups on the dimensions of social functioning and the relation with peers (Kidscreen-27 Social Support and 30 Peers, adjusted mean difference: $3 \cdot 25[95 \%$ CI $-0 \cdot 37$ to $6 \cdot 88], \mathrm{p}=0 \cdot 078 ; d 0 \cdot 63[0 \cdot 15$ to $1 \cdot 11])$.

The safety and tolerability of EGCG were good. Adverse events were mainly mild and not thought to be related to the treatment, with no differences between the EGCG and 35 placebo groups (table 2). There were no withdrawals related to drug tolerability. No significant differences were detected for changes in AST $(\mathrm{p}=0 \cdot 831)$ and ALT $(\mathrm{p}=0.623$; appendix $\mathrm{p} 12$ ), nor were there alterations in ECG.

6 months after treatment discontinuation, the positive 40 effects of EGCG and cognitive training on memory and executive functions persisted. The EGCG and cognitive training group had a higher memory performance in the immediate recall of verbal information in the list learning task (CRT free immediate recall, adjusted mean difference 451.98 points [ $95 \%$ CI 0.23 to 3.74 ], $\mathrm{p}=0.027 ; d 0.41$ $[-0.05$ to $0 \cdot 87])$. Also, a greater accuracy and response speed was detected in the EGCG and cognitive training group in the response inhibition task (Cats and Dogs total correct score, adjusted mean difference: $0.51[95 \% \mathrm{CI}$ 500.09 to 0.93 ], $\mathrm{p}=0.017 ; d 0.46$ [0.01 to 0.92 ]; Cats and Dogs total response time: $-5 \cdot 21 \mathrm{~s}[-8 \cdot 5$ to $-1 \cdot 92], \mathrm{p}=0 \cdot 002$; $d-0.6[-1.07$ to $-0 \cdot 12])$. The positive effect on adaptive behaviour on basic health care and safety skills (ABAS-II health and safety, adjusted mean difference: 2.9 [95\% CI $55-0 \cdot 25$ to $6 \cdot 05], \mathrm{p}=0 \cdot 071 ; d 0 \cdot 41[0 \cdot 03$ to $0 \cdot 85])$ in the group receiving EGCG and cognitive training was not significant (appendix pXX $\Omega$ he positive effects on ABAS-II 
functional academics score also faded after discontinuing the treatment (adjusted mean difference: $3 \cdot 22$ [95\% CI $-1 \cdot 44$ to $7 \cdot 88$ ], $p=0 \cdot 172$ ). Finally, we did not find significant effects on overall quality of life (Kidscreen-27 total score, adjusted mean difference: $10 \cdot 09$ [95\% CI $-5 \cdot 86$ to 26.04], $\mathrm{p}=0 \cdot 21)(\Omega$

Ten individuals in the EGCG and cognitive training group and ten in the placebo and cognitive training group had valid fMRI assessments at baseline. Eight patients in the placebo and cognitive training group and ten in the EGCG and cognitive training group had valid fMRI assessments both at baseline and 6 months after initiating treatment; and two of ten patients were excluded from the EGCG and cognitive training group because of motion artefacts. Nine patients in the placebo and cognitive training group and nine in the EGCG and cognitive training group had valid fMRI assessments both at baseline and 12 months; and one patient was excluded from each group because of motion artefacts. Thus, we report the results for 6 months and 12 months separately (appendix p 20, 21). fMRI session (baseline vs 6 months) by treatment interaction of the functional connectivity degree analysis, showed a significant interaction in the frontal cortex ( $\mathrm{t}$ score $=4 \cdot 2)$, Wernicke area (t score $=3 \cdot 1$ ), cerebellum (t score $=4 \cdot 2$; appendix pXX $\bigcirc d$ in a subcortical cluster partly involving a number of regions (appendix pXX $\_$post-hoc paired $t$ test showed that most of the entect was due to an increase in connectivity associated with the EGCG and cognitive training group (appendix p 19). For the seed- 30 based functional connectivity analysis, three different functional connectivity maps were generated using the areas that had significant effects in the connectivity degree analysis as the seed regions of interest. In the frontal cortex seed map, session-by-treatment interaction showed a significant increase in connectivity in the frontal (t score $=4 \cdot 7$ ), somatosensory (t score $=4 \cdot 2$ ), and occipito-temporal cortices ( $\mathrm{t}$ score $=4 \cdot 7$; appendix $\mathrm{p}$ 17). In the Wernicke seed map, we noted a significant interaction in the posterior cingulate cortex and the 40 precuneus (t score $=4 \cdot 0$ ). In the cerebellar seed map, significant interactions were noted within the cerebellum ( $t$ score $=5 \cdot 0$; appendix $p$ 17). After 12 months of treatment, we recorded changes (baseline vs 12 months) in the same areas identified at 6 months, suggesting a 45 persistent effect $\mathrm{EGCG}$ and cognitive training (appendix pXY $\bigcirc$ However, the changes were only significant in the frontal cortex (appendix pX)

The placebo and cognitive training group and the EGCG and cognitive training group had slightly different 50 ICF-15 baseline values, with the facilitation values in the placebo and cognitive training group being closer to normal than in the EGCG and cognitive training group (normal mean baseline ICF-15 value 1.22 [SD 0.40], placebo and cognitive training $2 \cdot 72[2 \cdot 36]$, EGCG and 5 cognitive training $3 \cdot 28[2 \cdot 19])$. At 6 months, the placebo and cognitive training group fared significantly better than the EGCG and cognitive training group (mean ICF$152 \cdot 31$ [SD 1.61] vs 3.47 [3.69], $\mathrm{p}=0 \cdot 015)$, although the median changes from baseline were not significant $(-0.41$ [IQR 1.12] vs 0.18 [2.72]). At 12 months of treatment, the increased facilitation was significantly abated in the group receiving EGCG and cognitive training compared with the placebo and cognitive training group (mean ICF-15 2.01 [1.43] vs 3.61 [5.81], $\mathrm{p}<0.0001$; median change from baseline -1.28 [IQR $1 \cdot 58]$ vs 0.89 [2.10], $\mathrm{p}<0 \cdot 0001)$. No significant differences were detected for ICI- $3(\mathrm{p}=0 \cdot 67)$, ICI- $5(\mathrm{p}=0 \cdot 67)$, or ICF$10(\mathrm{p}=0 \cdot 56$; appendix p 23).

In the EGCG and cognitive training group at 12 months, we noted significant changes from baseline in total cholesterol (adjusted mean difference -8.91 [95\% CI $-16 \cdot 3$ to $-1 \cdot 51$ ], $\mathrm{p}=0 \cdot 019$; figure 3 ), oxidised LDL (adjusted mean difference $-3 \cdot 7[-6.93$ to $-0 \cdot 44], \mathrm{p}=0.027)$ and HDL (adjusted mean difference $-4.06[-7 \cdot 39$ to $-0 \cdot 72], \mathrm{p}=0 \cdot 011$ ) plasma concentrations, that were reduced in the EGCG and cognitive training group compared with the placebo and cognitive training group (appendix $p$ 11). No significant differences were detected for LDL $(p=0 \cdot 072)$ and triglycerides $(\mathrm{p}=0 \cdot 178$; appendix $\mathrm{p} 11)$.

We also recorded significant increases from baseline in 5 total plasma homocysteine in the group receiving EGCG and cognitive training compared with the placebo and cognitive training group after the 12 months of intervention (adjusted mean difference 0.7 [95\% CI $0 \cdot 14$ to $1 \cdot 26$, $\mathrm{p}=0 \cdot 015$; figure 3 ). The differences in homocysteine plasma concentrations disappeared after discontinuing treatment with EGCG (adjusted mean difference $0 \cdot 15$ [95\% CI $-0 \cdot 64$ to $0 \cdot 93$ ], $\mathrm{p}=0 \cdot 71)$. Transthyretin results are shown in the appendix (p 11).

After 12 months of treatment, our analyses did not show significant weight loss or change in body-mass index (BMI) (appendix p 11). At 18 months, 6 months after

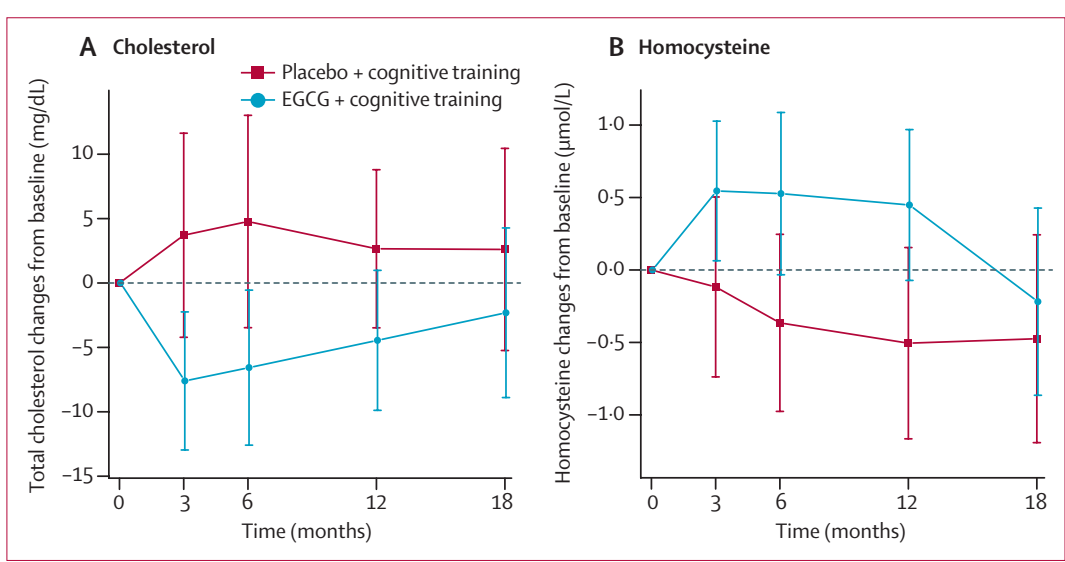

Figure 3: Treatment effects of EGCG and cognitive training versus placebo and cognitive training on total cholesterol and total homocysteine plasma concentrations

(A) Treatment effects of EGCG and cognitive training on total cholesterol were significant at 12 months ( $\mathrm{p}=0.019)$ but were no longer significant after discontinuing EGCG at 18 months $(p=0.506)$. (B) Treatment effects of EGCG and cognitive training on total homocysteine were significant at 12 months $(p=0.015)$ but were no longer significant after discontinuing EGCG at 18 months ( $p=0 \cdot 71)$. EGCG=epigallocatechin-3-gallate. 


\begin{tabular}{|lll|}
\hline & $\begin{array}{l}\text { Placebo and } \\
\text { cognitive } \\
\text { training }(\mathrm{n}=43)\end{array}$ & $\begin{array}{l}\text { EGCG and } \\
\text { cognitive } \\
\text { training }(\mathrm{n}=41)\end{array}$ \\
\hline Serious adverse events & $1(2 \%)$ & $2(5 \%)$ \\
Mental disorder & $1(2 \%)$ & $1(2 \%)$ \\
Admission to hospital & & \\
Non-serious adverse events & $21(42 \%)$ & $18(42 \%)$ \\
Upper respiratory tract infection & $5(10 \%)$ & $6(14 \%)$ \\
Osteoarticular pain & $1(2 \%)$ & $3(7 \%)$ \\
Headache & $3(6 \%)$ & $3(7 \%)$ \\
Menstrual disturbances & $5(10 \%)$ & $3(7 \%)$ \\
Mood disorders & $5(10 \%)$ & $2(5 \%)$ \\
Abdominal pain & $4(8 \%)$ & $1(2 \%)$ \\
Skin infections & 0 & $2(5 \%)$ \\
Asthenia & 0 & $2(5 \%)$ \\
Celiac disease diagnosis & $2(4 \%)$ & 0 \\
Gastrointestinal infections & $2(4 \%)$ & 0 \\
Tremors and dizziness & & \\
Values are $n$ (\%). & & \\
\hline Table 2: Adverse events by treatment group occurring in more than two & \\
participants & & 10 \\
\hline
\end{tabular}

treatment discontinuation, differences in weight loss 2 between EGCG plus cognitive training and placebo plus cognitive training groups increased $(1.48 \mathrm{~kg})$ and were significant $(\mathrm{p}=0 \cdot 044)$, the mean difference from baseline being $-0.16 \mathrm{~kg}$ in the EGCG and cognitive training group versus $1.32 \mathrm{~kg}$ in the placebo and cognitive training group with similar differences in BMI ( $\mathrm{p}=0$-037; appendix $\mathrm{p} 11)$.

\section{Discussion}

Our study showed that patients in the EGCG plus cognitive training group performed better than those in the placebo plus cognitive training group in some cognitive tests and in adaptive behaviour after the 12 months of treatment (appendix pX $P$ he EGCG and cognitive training group had better preservation of recognition memory tasks and improvement in executive 4 function than the placebo and cognitive training group. Improvements in memory and executive function were accompanied by improved performance of daily tasks requiring basic literacy in the EGCG and cognitive training group. Benefits in functional academics imply an improvement in the use of basic literacy skills (reading, writing, and mathematics), allowing daily independent functioning (ie, recognise the time, be able to read and write small notes, recognise small quantities when paying, and performing small operations). Sustained effects of EGCG were observed after 18 months in memory and executive function. Even though the effects of EGCG and cognitive training on cognitive function were small and of subclinical magnitude, they were accompanied by a positive functional change on adaptive behaviour, with an absence of relevant negative side-effects. Safety monitoring showed no adverse effects
1 associated with EGCG and cognitive training. The benefits seem thus substantial, given the fairly short treatment (1 year).

The improvement in memory and executive function 5 deficits with EGCG and cognitive training were similar to the results of our previous phase 1 clinical trial ${ }^{9}$ using EGCG without cognitive training. In both trials, the main effect of EGCG was the improvement of immediate recognition memory. This measure is sensitive to 10 hippocampal and, in particular, perirhinal cortex activity, in addition to regions such as ventromedial cortices. ${ }^{14}$ In Down's syndrome, altered functionality of the hippocampus and prefrontal cortex contributes to memory and executive functioning deficits ${ }^{15,16}$ and, in a 15 previous neuroimaging study, ${ }^{12}$ we identified distinct connectivity disturbances in frontal and anterior temporal structures.

Our study was not powered to assess the effect of the treatment on each component of the secondary outcomes. 20 Nonetheless, EGCG and cognitive training was superior to placebo in two secondary outcome components, with a significant increase in connectivity in the frontal, somatosensory, and occipito-temporal cortices, and cortical excitability normalisation. Resting-state fMRI measurements showed a marked enhancement of regional functional connectivity with increases in the functional integration of cortical and subcortical distributed networks, including the frontal cortex, Wernicke area, the precuneus, occipito-temporal and somatosensory cortices, and basal ganglia in the EGCG and cognitive training group. Treatment-related changes in functional connectivity within frontal networks were significantly correlated with the increase in ABAS-II functional academic skills. Using non-invasive TMS, ${ }^{17-19}$ we showed that EGCG and cognitive training normalised intracortical facilitation. We speculate that these effects could also contribute to the improved cognitive abilities in individuals treated with EGCG and cognitive training, since cognitive deficits in Down's syndrome have been proposed to result from an imbalance, with an excess of synaptic inhibition in the hippocampus and increased excitation in the cerebral cortex. ${ }^{18,20}$

In previous studies, ${ }^{8,9}$ the efficacy of EGCG was suggested to depend, at least partly, on the ability of EGCG to inhibit DYRK1A kinase activity, a major candidate for Down's syndrome cognitive and neural plasticity phenotypes. ${ }^{1,2}$ Total homocysteine plasma concentrations, used as a surrogate biomarker of DYRK1A kinase activity, ${ }^{21,22}$ were increased to amounts that were not in the cardiovascular risk range in the EGCG and cognitive training group but not in the placebo and cognitive training group, and returned to baseline concentrations after treatment discontinuation. However, we did not control for other factors such as vitamin B12 or folate that could also contribute to increased homocysteine plasma concentrations. In this study, we also noted a reduction in total cholesterol and oxidised LDL concentrations. Thus, a 
lipid-lowering effect combined with a reduced lipid oxidation might contribute to the therapeutic effect. However, other mechanisms of action of EGCG should not be disregarded, including epigenetic, ${ }^{23}$ mitochondrial dysfunction rescue, ${ }^{24}$ antioxidant, and hypolipidaemic effects. ${ }^{25}$

Markers of cardiac and hepatic toxicity were endpoints of particular interest. In-vitro studies have shown that EGCG is a low-affinity inhibitor of hERG ${ }^{26}$ but in-vivo studies in dogs could not relate these effects to cardiac toxicity. ${ }^{27}$ Nevertheless, cardiac toxicity at high doses cannot be excluded and caution should be taken when EGCG is given concurrently with cardiovascular drugs. In our study, we did not see alterations in ECG during EGCG treatment. Markers of hepatotoxicity were unaltered throughout the study. The pharmacokinetics and metabolic disposition of EGCG have been reported; ${ }^{28}$ clinical studies with EGCG in multiple sclerosis at doses in the range of those tested in the present study and for similar periods of exposure showed that EGCG plasma concentrations were associated with changes in brain $\mathrm{N}$-acetyl aspartate, an EGCG metabolite. ${ }^{29}$

The study had some limitations. First, for technical reasons we could not measure amyloidosis biomarkers. We do not believe this limitation compromises the 25 conclusions of our study because our population was within an age range in which neurodegeneration due to Alzheimer's disease is unlikely, and we monitored clinical signs with specific questionnaires like the Dementia Questionnaire for Persons with Mental 30 Retardation. Second, although our study had a long follow-up, phase 3 trials with a larger population of individuals with Down's syndrome will be needed to assess and confirm the long-term efficacy of EGCG and cognitive training. We do not expect serious side-effects if the EGCG dose is maintained at fairly low concentrations such as those used in our study. Third, due to the large number of tests done in the framework of the regression models, the family-wise error rate exceeded $0 \cdot 05$. Nonetheless, to protect against type II errors, no corrections for multiple comparisons were applied. Otherwise, with a family-wise error rate of $0 \cdot 05$, the significance of each test would have been restrictive. No significant positive cognitive or functional effects were detected in the placebo group. To rule out the possibility that any of the significant results was a type I error, further studies are needed. Fourth, we are aware that the choice of our assessment instruments might have restricted the detection of EGCG effects. Therefore, this battery will undergo future modifications to integrate 50 the feedback from the TESDAD study, future clinical trials, and any clinical or preclinical evidence. Nevertheless, the state of the field in testing for cognitive changes is not yet appropriately developed and findings from our clinical trial cannot be validated against a 5 consensus gold standard. This is an important caveat that should be considered when interpreting the results of this study. Finally, although it is not possible to compare EGCG with other treatments, we expect to see more developments in this area of research, especially in multicomponent therapies. However, it should be noted that the long-term efficacy of cognitive training has not been conclusively shown in people with Down's syndrome across all studies.

We conclude that EGCG with cognitive training had beneficial effects on memory and executive deficits with enhancement of the everyday life competence in young adults with Down's syndrome, although some domains were not significantly modified by the treatment. The combined intervention of EGCG and cognitive training for 12 months seem to ameliorate functional integration of cortical and subcortical brain networks in this population. Although other statistically underpowered studies have reported preliminary data suggesting that cognitive and adaptive skills in Down's syndrome might be treated through pharmacological intervention, ${ }^{9,30}$ this study is the first well-powered trial that shows improvement in adaptive behaviour (functional academics) and brain-related changes in young adults with Down's syndrome. However, more research is needed to clarify the nature of the beneficial association between the EGCG and cognitive training intervention (ie, synergistic or additive). Although efficacy combined with a good safety profile supports the use of this treatment in clinics, more phase 2 and 3 clinical trials are warranted to further support the use of this catechin for improvement of cognitive function in Down's syndrome.

Contributors

RdlT and MD designed the study, raised funding, analysed data, and wrote the manuscript. HB participated in the design of the study. JP and LB-H did the neuroimaging studies, analysed data, and contributed to the writing of the manuscript. JME and AP did the neurophysiological studies and contributed to the writing of the manuscript. SdS, AC-R, SC-S, GS-B, LdH, and BB conceived and did the validation of the TESDAD neuropsychological test battery, did the cognitive and functional investigations, analysed data, and contributed to the writing of the manuscript. MF, GH, and ID-E participated in the medical assessments, safety evaluation of treatments, data analysis, 0 and contributed to the writing of the manuscript. JR, KL, and SV participated in the coordination of the study, statistical analysis, and contributed to the writing of the manuscript. LX, JMD, NJ, and MF participated in the collection of biological samples, biomarker analysis, data analysis, and contributed to the writing of the manuscript.

\section{TESDAD study group}

5 Aida Cuenca-Royo (site investigator; Hospital del Mar Medical Research Institute, Barcelona, Spain), Alessandro Principe (site investigator; Neurophysiology Section, Hospital del Mar Medical Research Institute), Bessy Benejam (site investigator; Catalan Foundation of Down Syndrome, FCSD, Barcelona, Spain), Ester Civit (site investigator; Hospital del Mar Medical Research Institute, Barcelona, Spain), Gimena Hernandez (site investigator; Hospital del Mar Medical Research Institute, Barcelona, Spain), Gonzalo Sanchez (site investigator; Hospital del Mar Medical Research Institute, Barcelona, Spain), Henri Bléhaut (site investigator; Jérôme Lejeune Foundation, Paris, France), Ivan Dueñas-Espín (site investigator; Hospital del Mar Medical Research Institute, Barcelona, Spain), Jesús Pujol (site investigator; MRI Unit, Hospital del Mar Medical Research Institute, Barcelona, Spain), Joan Rodriguez (study coordinator; Hospital del Mar Medical Research Institute, Barcelona, Spain), Josep $\mathrm{M}^{\mathrm{a}}$ Espadaler (site 
investigator; Neurophysiology Section, Hospital del Mar Medical Research Institute, Barcelona, Spain), Judit Sánchez-Gutiérrez (site investigator; Feskits, Barcelona, Spain), Katy Trias (site investigator; FCSD, Barcelona, Spain), Klaus Langohr (statistician; Polytechnics University, Barcelona, Spain), Laia Roca (study manager; Hospital del Mar Medical Research Institute, Barcelona, Spain), Laura Blanco-Hinojo 5 (site investigator; Hospital del Mar Medical Research Institute, Barcelona, Spain), Laura del Hoyo (site investigator; Hospital del Mar Medical Research Institute, Barcelona, Spain), Laura Xicota (site investigator; Hospital del Mar Medical Research Institute, Barcelona, Spain), Magí Farré (co-principal investigator; Hospital del Mar Medical Research Institute, Barcelona, Spain), Mara Dierssen (co-principal investigator; Centre for Genomic Regulation, Barcelona, Spain), Rafael de la Torre (co-principal investigator; Hospital del Mar Medical Research Institute, Barcelona, Spain), Rut Freixas (site investigator; CRG, Barcelona, Spain), Sebastià Videla (site investigator; FCSD, Barcelona, Spain), Montserrat Fitó (site investigator; Hospital del Mar Medical Research Institute, Barcelona, Spain), Silvina Catuara-Solarz (site investigator; CRG, Barcelona, Spain), Susana de Sola (site investigator; Hospital del Mar Medical Research Institute, Barcelona, Spain), Valérie Legout (site investigator; Jérôme Lejeune Foundation, Paris, France), and Jean Maurice Delabar (site investigator; Université Paris Diderot, Sorbonne, Paris, France).

\section{Declaration of interests}

We declare no competing interests.

\section{Acknowledgments}

We acknowledge the support of Jérôme Lejeune Foundation, Instituto de Salud Carlos III FEDER (PI11/ 00744), MINECO (SAF2010-19434 and SAF2013-49129-C2-1-R, and PCIN-2013-060), and Generalitat de Catalunya (SGR 2009/1450, SGR 2009/718, SGR 2014/680, and SGR 2014/1125). Center for Genomic Regulation is a Center of Excellence Severo Ochoa SEV-2012-0208. We are in debt to the families that participated in the study and in particular to the contribution of the Catalan Down Syndrome Foundation (Barcelona Spain) and Down España (Madrid, Spain) that made the study possible. We acknowledge the collaboration of Life Extension in the preparation of medication, and of Fundació Espai Salut for the provision for research purposes of the telematics platform Feskits

\section{References}

1 de la Torre R, Dierssen M. Therapeutic approaches in the improvement of cognitive performance in Down syndrome: past, present, and future. Prog Brain Res 2012; 197: 1-14.

2 Dierssen M. Down syndrome: the brain in trisomic mode. Nat Rev Neurosci 2012; 13: 844-58.

3 Couzens D, Haynes M, Cuskelly M. Individual and environmental characteristics associated with cognitive development in Down syndrome: a longitudinal study. J Appl Res Intellect Disabil 2012; 25: 396-413.

For more on Life Extension see http://www.lifeextension.com

For more on Fundació Espai Salut see http://www.espaisalut.

4 Connolly BH, Morgan SB, Russell FF, Fulliton WL. A longitudinal study of children with Down syndrome who experienced early intervention programming. Phys Ther 1993; 73: 170-79.

5 Rendeiro C, Rhodes JS, Spencer JP. The mechanisms of action of flavonoids in the brain: direct versus indirect effects. Neurochem Int 2015; 89: 126-39.

6 Bain J, McLauchlan H, Elliott M, Cohen P. The specificities of protein kinase inhibitors: an update. Biochem J 2003; 371: 199-204.

7 Lin LC, Wang MN, Tseng TY, Sung JS, Tsai TH. Pharmacokinetics of (-)-epigallocatechin-3-gallate in conscious and freely moving rats and its brain regional distribution. J Agric Food Chem 2007; 55: 1517-24.

8 Guedj F, Sebrie C, Rivals I, et al. Green tea polyphenols rescue of brain defects induced by overexpression of DYRK1A. PLoS One 2009; 4: e4606

9 de la Torre R, De Sola S, Pons M, et al. Epigallocatechin-3-gallate, a DYRK1A inhibitor, rescues cognitive deficits in Down syndrome mouse models and in humans. Mol Nutr Food Res 2014; 58: 278-88.

10 Pons-Espinal M, Martinez de LM, Dierssen M. Environmental enrichment rescues DYRK1A activity and hippocampal adult neurogenesis in TgDyrk1A. Neurobiol Dis 2013; 60: 18-31.

11 De Sola S, de la Torre R, Sanchez-Benavides G, et al. A new cognitive evaluation battery for Down syndrome and its relevance for clinical trials. Front Psychol 2015; 6: 708.
112 Pujol J, del Hoyo L, Blanco-Hinojo L, et al. Anomalous brain functional connectivity contributing to poor adaptive behavior in Down syndrome. Cortex 2015; 64: 148-56.

13 Pujol J, Macia D, Blanco-Hinojo L, et al. Does motion-related brain functional connectivity reflect both artifacts and genuine neural activity? Neuroimage 2014; 101: 87-95.

14 Buffalo EA, Bellgowan PS, Martin A. Distinct roles for medial temporal lobe structures in memory for objects and their locations. Learn Mem 2006; 13: 638-43.

15 Costanzo F, Varuzza C, Menghini D, et al. Executive functions in intellectual disabilities: a comparison between Williams syndrome and Down syndrome. Res Dev Disabil 2013; 34: 1770-80.

1016 Hanna-Pladdy B. Dysexecutive syndromes in neurologic disease. J Neurol Phys Ther 2007; 31: 119-27.

17 Nardone R, Marth R, Ausserer H, Bratti A, Tezzon F. Reduced short latency afferent inhibition in patients with Down syndrome and Alzheimer-type dementia. Clin Neurophysiol 2006; 117: 2204-10.

18 Oberman L, Ifert-Miller F, Najib U, et al. Transcranial magnetic stimulation provides means to assess cortical plasticity and excitability in humans with fragile $\mathrm{X}$ syndrome and autism spectrum disorder. Front Synaptic Neurosci 2010; 2: 26.

19 Olazaran J, Prieto J, Cruz I, Esteban A. Cortical excitability in very mild Alzheimer's disease: a long-term follow-up study. J Neurol 2010; 257: 2078-85.

20 Thomazeau A, Lassalle O, Iafrati J, et al. Prefrontal deficits in a murine model overexpressing the down syndrome candidate gene dyrk1a. J Neurosci 2014; 34: 1138-47.

21 Noll C, Planque C, Ripoll C, et al. DYRK1A, a novel determinant of the methionine-homocysteine cycle in different mouse models overexpressing this Down-syndrome-associated kinase. PLoS One 2009; 4: e7540.

2522 Noll C, Tlili A, Ripoll C, et al. Dyrk1a activates antioxidant NQO1 expression through an ERK1/2-Nrf2 dependent mechanism. Mol Genet Metab 2012; 105: 484-88.

23 Ramakrishna N, Meeker HC, Brown WT. Novel epigenetic regulation of alpha-synuclein expression in Down syndrome. Mol Neurobiol 2016; 53: 155-62.

24 Vacca RA, Valenti D. Green tea EGCG plus fish oil omega-3 dietary supplements rescue mitochondrial dysfunctions and are safe in a Down's syndrome child. Clin Nutr 2015; 34: 783-84.

25 Lee SJ, Jia Y. The effect of bioactive compounds in tea on lipid metabolism and obesity through regulation of peroxisome proliferator-activated receptors. Curr Opin Lipidol 2015; 26: 3-9.

26 Kelemen K, Kiesecker C, Zitron E, et al. Green tea flavonoid

35 epigallocatechin-3-gallate (EGCG) inhibits cardiac hERG potassium channels. Biochem Biophys Res Commun 2007; 364: 429-35.

27 Kapetanovic IM, Crowell JA, Krishnaraj R, et al. Exposure and toxicity of green tea polyphenols in fasted and non-fasted dogs. Toxicology 2009; 260: 28-36.

28 Feng WY. Metabolism of green tea catechins: an overview. Curr Drug Metab 2006; 7: 755-809.

29 Lovera J, Ramos A, Devier D, et al. Polyphenon E, non-futile at neuroprotection in multiple sclerosis but unpredictably hepatotoxic: phase I single group and phase II randomized placebo-controlled studies. J Neurol Sci 2015; 358: 46-52.

30 Boada R, Hutaff-Lee C, Schrader A, et al. Antagonism of NMDA receptors as a potential treatment for Down syndrome: a pilot randomized controlled trial. Transl Psychiatry 2012; 2: e141. 